\title{
Robust Dissipative Control for Networked Control Systems with Multiple Packet Dropouts *
}

\author{
Jia Wang, Qing-Long Han*, Fuwen Yang \\ Centre for Intelligent and Networked Systems \\ School of Information and Communication Technology \\ Central Queensland University, Rockhampton QLD 4702, Australia \\ *Corresponding author: Tel. +61 74930 9270; \\ Fax +61749309729 \\ E-mail: $\{j . w a n g, q . h a n\} @ c q u . e d u . a u$
}

\begin{abstract}
This paper deals with the dissipative problem for uncertain time-delay networked control systems with both multiple measurement and control packet dropouts. The uncertainty is assumed to satisfy a dissipative inequality, and the multiple measurement and control packet dropouts are described by two independent Bernoulli distributed sequences. By utilizing the Lyapunov functional method, a robust dissipative controller is designed such that the corresponding closed-loop system is asymptotically mean-square stable and strict $(Q, S, R)$ dissipative. The sufficient condition on the existence of the controller is formulated in the form of linear matrix inequalities. Then the controller gain is achieved by using an extended cone complementarity linearization method. An example is given to illustrate the effectiveness of the proposed design method.
\end{abstract}

Keywords: Robust dissipative control, networked control systems, multiple packet dropouts.

\section{INTRODUCTION}

Networked control systems (NCSs) are spatially distributed systems in which the communication between sensors, actuators and controllers occurs through a shared bandlimited communication network (Hespanha et al. (2007)). The primary advantages of NCSs are reduced system wiring, ease of system diagnosis and maintenance, and increased system agility (Zhang et al. (2001)). Therefore, increasing research attention has recently been paid to the study of NCSs, see for example, Yue et al. (2004) and $\mathrm{Yu}$ et al. (2004). However, since sensors, actuators and controllers are connected via a shared band-limited communication network, network-induced delays and packet dropouts in NCSs are inevitable. The existence of the network-induced delays and packet dropouts may degrade the performance of the control system or even drive the system to instability. Therefore, a realistic networked control system design should take the network-induced delays and the packet dropouts into account. In the past two decades, the problems of network-induced delays and packet dropouts in the networked control systems have been extensively studied. For example, Yue et al. (2004) indicated the state feedback stabilization of networked systems with packet dropouts and $\mathrm{Yu}$ et al. (2004) discussed the packet dropout and transmission delays in networked

* This work was supported in part by the Australian Research Council Discovery Projects under Grant DP1096780 and Grant DP0986376; the Research Advancement Awards Scheme Program (January 2010 - December 2012) and the RDI Merit Grant Scheme Project under Gant RDIM1109 (January 2011 - December 2011) at Central Queensland University, Australia. control systems in both continuous-time case and discretetime case using LMI approach.

Since the introduction of dissipativity by Willems (1972), the dissipative problem for variety of practical systems has been attracting much attention. Dissipativity is a generation of passivity in electrical networks and other dynamic systems with dissipative energy. Dissipative property is an expected system behavior, since the storage function is closely related to system energy and serves as a candidate for a Lyapunov function. When the dissipativity is assured, stability problems can be solved. Therefore, it has been successfully used in diverse areas such as systems, circuits, robots and control theory and applications. For instance, Xie et al. (1998) discussed robust dissipative control for linear systems and Li et al. (2002) extended this work to the delay-dependant quadratic dissipative control. Similarly, Yang et al. (2007) suggested the dissipative control of singular systems and Shen et al. (1999) analyzed the dissipative theory approach for robot systems. The reliable dissipative control for stochastic impulsive systems was considered by Zhang et al. (2008). As discussed already, dissipative control has been investigated by many researchers and many results have been reported in the literature. Since energy exists in NCSs and it may change during transmission, it is not surprising that the dissipativity theory is generalized to the NCSs. Very recently, the passivity and passification problems were discussed by Gao et al. (2007). However, to the best of the authors' knowledge, for the networked control systems, in the simultaneous presence of time-delays, packet dropouts and uncertainties, the problem of dissipative control has 
received less research attention to date and still remains challenging, which motivates the present study.

Based on the above discussions, the object of this paper is to deal with the problem of dissipative output feedback controller design for a class of networked control systems with multiple packet dropouts, where the plant is a timedelay system. First, we will extend the definition of dissipativity to the stochastic setting. By using Lyapunov approach and stochastic analysis method, the dissipativity performance condition in the sense of expectation will be formulated in the forms of linear matrix inequalities. Then robust dissipative controller will be derived, which guarantees the closed-loop system is asymptotically mean-square stable and strictly mean-square $(Q, S, R)$-dissipative. The extended cone complementarity linearization method will be proposed to pursue a suboptimal system performance. A numerical example will be provided to illustrate the effectiveness of the proposed method.

\section{PROBLEM FORMULATION}

Consider the following system with time-delay

$$
\left\{\begin{aligned}
x(k+1)= & A x(k)+A_{d} x(k-d)+\sum_{i=1}^{p} H_{1 i} \xi_{i}(k) \\
& +B_{1} w(k)+B_{2} u^{F}(k), \\
z(k)= & C_{1} x(k)+C_{d} x(k-d)+\sum_{i=1}^{p} H_{2 i} \xi_{i}(k) \\
& +D_{11} w(k)+D_{12} u^{F}(k), \\
y(k)= & C_{2} x(k)+D_{21} w(k), \\
\sigma_{i}(k)= & E_{1 i} x(k)+E_{d i} x(k-d) \\
& +E_{2 i} w(k)+E_{3 i} \xi(k)+E_{c} u^{F}(k), \\
x(k)= & \phi(k), \quad k=-d,-d+1, \ldots,-1,0,
\end{aligned}\right.
$$

where $x(k) \in \mathbb{R}^{n}$ is the state vector, $w(k) \in \mathbb{R}^{r}$ is the disturbance belonging to $l_{2}[0, \infty), z(k) \in \mathbb{R}^{q}$ is the output, $u^{F}(k) \in \mathbb{R}^{m}$ is the control input, $y(k) \in$ $\mathbb{R}^{p}$ is the measure output vector; $d>0$ is a known time delay, $\phi(k)$ represents the initial function; Matrices $A, B_{1}, B_{2}, C_{1}, C_{2}, D_{1}, D_{2}, H_{1 i}, H_{2 i}, H_{3 i}, E_{1 i}, E_{2 i}, E_{3 i}, E_{4 i}$ are known real matrices with appropriate dimensions.

The variables $\xi_{i}(k) \in \mathbb{R}^{k_{i}}$ and $\sigma_{i}(k) \in \mathbb{R}^{h_{i}}$, which are used to describe the system uncertainty, are assumed to satisfy the following dissipative inequality

$$
\xi_{i}^{T}(k) L_{i} \xi_{i}(k)+2 \xi_{i}^{T}(k) M_{i} \sigma_{i}(k)+\sigma_{i}^{T}(k) G_{i} \sigma_{i}(k) \geq 0
$$

where $L_{i}, M_{i}$, and $G_{i}$ are known real constant matrices with $L_{i}$ and $G_{i}$ are symmetric.

$$
\begin{aligned}
& \bar{L}=\operatorname{diag}\left\{L_{1}, L_{2}, \ldots, L_{p}\right\}, \quad H_{1}=\left[\begin{array}{llll}
H_{11} & H_{12} & \ldots & H_{1 p}
\end{array}\right], \\
& \bar{M}=\operatorname{diag}\left\{M_{1}, M_{2}, \ldots, M_{p}\right\}, H_{2}=\left[\begin{array}{llll}
H_{21} & H_{22} & \ldots & H_{2 p}
\end{array}\right] \text {, } \\
& \bar{G}=\operatorname{diag}\left\{I_{1}, I_{2}, \ldots, I_{p}\right\}, \quad H_{3}=\left[\begin{array}{llll}
H_{31} & H_{32} & \ldots & H_{3 p}
\end{array}\right], \\
& E_{1}^{T}=\left[\begin{array}{llll}
E_{11}^{T} & E_{12}^{T} & \ldots & E_{1 p}^{T}
\end{array}\right]^{T}, \quad E_{2}^{T}=\left[\begin{array}{llll}
E_{21}^{T} & E_{22}^{T} & \ldots & E_{2 p}^{T}
\end{array}\right]^{T} \text {, } \\
& E_{3}^{T}=\left[\begin{array}{llll}
E_{31}^{T} & E_{32}^{T} & \ldots & E_{3 p}^{T}
\end{array}\right]^{T}, \\
& \xi(k)=\left[\begin{array}{lllll}
\xi_{1}^{T}(k) & \xi_{2}^{T}(k) & \ldots & \xi_{p}^{T}(k)
\end{array}\right]^{T}, \\
& \sigma(k)=\left[\begin{array}{lllll}
\sigma_{1}^{T}(k) & \sigma_{2}^{T}(k) & \ldots & \sigma_{p}^{T}(k)
\end{array}\right]^{T} .
\end{aligned}
$$

Then from the dissipative inequality (2), we have

$$
\left[\xi^{T}(k) \sigma^{T}(k)\right]^{T}\left[\begin{array}{cc}
\bar{L} & \bar{M}^{T} \\
\bar{M} & \bar{G}
\end{array}\right]\left[\begin{array}{l}
\xi(k) \\
\sigma(k)
\end{array}\right] \geq 0
$$

Throughout this paper, suppose that the packet transfers between the controller and the remote system, e.g., sensors and actuators in a distributed control system, in the presence of the control network, which is shown in Fig.1.

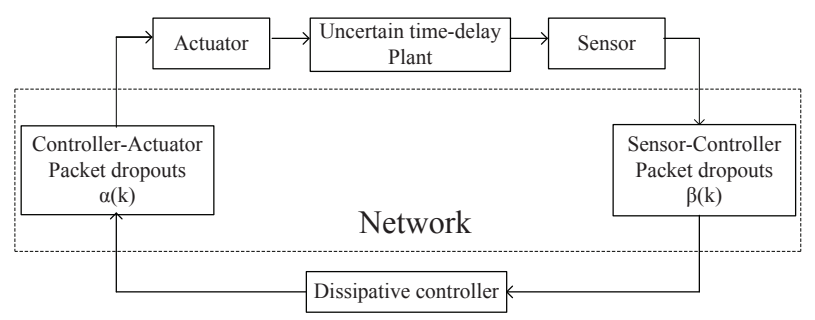

Fig. 1. Structure of a networked control system.

Because network is introduced to control- system design, the packet dropouts phenomenon, which appears in a typical network environment, may make the controller-design problem much more involved. Considering the multiple packet dropouts, the network can be modeled as a switch that opens and closes in a random manner. When the switch is open, its output at the previous value, which means the packet is lost. The measure signals are sent by the plant to the controller via the communication channel and there may be random dropouts, where only the probability of the dropouts is known. The current observation $y^{F}(k)$ is the current system output $y(k)$, with the probability of $(1-\beta(k))$. Similar to the measurement channel, the control signals sent by the remote controller to the plant via the communication channel. The plant control input $u^{F}(k)$ is the current controller output $u(k)$, with the probability of $(1-\alpha(k))$. The multiple control and measurement packet dropouts can be represented by the following relations

$$
\left\{\begin{array}{l}
u^{F}(k)=(1-\alpha(k)) u(k)+\alpha(k) u^{F}(k-1) \\
y^{F}(k)=(1-\beta(k)) y(k)+\beta(k) y^{F}(k-1)
\end{array}\right.
$$

Both $\alpha(k)$ and $\beta(k)$ are satisfied with the Bernoulli distributed white sequence

$$
\left\{\begin{array}{l}
\operatorname{Prob}\{\alpha(k)=1\}=\mathbf{E}\{\alpha(k)\}:=\bar{\alpha}, \\
\operatorname{Prob}\{\alpha(k)=0\}=1-\mathbf{E}\{\alpha(k)\}:=1-\bar{\alpha}, \\
\operatorname{Prob}\{\beta(k)=1\}=\mathbf{E}\{\beta(k)\}:=\bar{\beta}, \\
\operatorname{Prob}\{\beta(k)=0\}=1-\mathbf{E}\{\beta(k)\}:=1-\bar{\beta}
\end{array}\right.
$$

where $\operatorname{Prob}\{\cdot\}$ means the occurrence probability of the event $\cdot \mathbf{E}\{\alpha(k)\}$ stands for the expectation of the stochastic variable $\alpha(k)$. $\mathbf{E}\{\beta(k)\}$ stands for the expectation of the stochastic variable $\beta(k) . \bar{\alpha}$ and $\bar{\beta}$ are known scalars. At the same time, $\alpha(k)$ and $\beta(k)$ are independent.

In this paper, we propose the following dynamic feedback controller for system (1)

$$
\left\{\begin{aligned}
\hat{x}(k+1) & =A_{F} \hat{x}(k)+B_{F} y^{F}(k) \\
u(k) & =C_{F} \hat{x}(k)
\end{aligned}\right.
$$

where $\hat{x}(k) \in \mathbb{R}^{n}$ is the state of the controller, $A_{F}, B_{F}, C_{F}$ are the parameters of the controller to be designed.

From the system (1) and controller (6), the closed-loop system can be described as

$$
\left\{\begin{aligned}
\eta(k+1) & =A_{c l} \eta(k)+\hat{A}_{d} Z \eta(k-d)+B_{c l} w(k)+H_{c l} \xi(k), \\
z(k) & =C_{c l} \eta(k)+C_{d} Z \eta(k-d)+D_{11} w(k)+H_{2} \xi(k), \\
\sigma(k) & =E_{c l} \eta(k)+E_{d} Z \eta(k-d)+E_{2} w(k)+E_{3} \xi(k) .
\end{aligned}\right.
$$

where 


$$
\begin{aligned}
& \eta(k)=\left[x(k)^{T} u^{F}(k-1)^{T} y^{F}(k-1)^{T} \hat{x}(k)^{T}\right]^{T}, \\
& \alpha \overline{(k)}=1-\alpha(k), \quad \beta \overline{(k)}=1-\beta(k), \quad H_{c l}=\left[\begin{array}{llll}
H_{1}^{T} & 0 & 0 & 0
\end{array}\right]^{T} \text {, } \\
& \hat{A}_{d}^{T}=\left[\begin{array}{llll}
A_{d}^{T} & 0 & 0 & 0
\end{array}\right], \quad Z=\left[\begin{array}{llll}
I & 0 & 0 & 0
\end{array}\right], \\
& A_{c l}=\left[\begin{array}{cccc}
A & \alpha(k) B_{2} & 0 & \alpha \overline{(k)} B_{2} C_{F} \\
0 & \alpha(k) I & 0 & \alpha \overline{(k)} C_{F} \\
\beta \overline{(k)} C_{2} & 0 & \beta(k) I & 0 \\
\beta \overline{(k)} B_{F} C_{2} & 0 & \beta(k) B_{F} & A_{F}
\end{array}\right],
\end{aligned}
$$

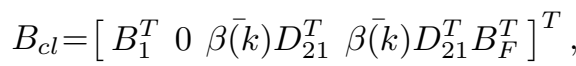

$$
\begin{aligned}
& C_{c l}=\left[\begin{array}{lllll}
C_{1} & \alpha(k) D_{12} & 0 & \alpha \overline{(k)} D_{12} C_{F}
\end{array}\right], \\
& E_{c l}=\left[\begin{array}{lllll}
E_{1} & \alpha(k) E_{c} & 0 & \alpha(k) & E_{c} C_{F}
\end{array}\right] .
\end{aligned}
$$

We are now ready to introduce the notion of dissipativity for the closed-loop system (7). In the literature, there have been several different definitions about dissipativity. In consideration of the presence of the network, we define the dissipativity in the sense of the expectation, which is an extension of the concept in Lozano et al. (2000). Definition 1. A function $E(w, z, T)$ is called a supply rate, if it satisfies

$$
\sum_{k=t_{1}}^{t_{2}} E(w, z, k)<\infty, t_{2}>t_{1} \geq 0
$$

Definition 2. The closed-loop system (7) is dissipative with respect to the supply rate $E(w, z, T)$ if there exists a nonnegative-definite function $V(x(t))$, called a storage function, such that the dissipation inequality

$$
V\left(x\left(t_{2}\right)\right)-V\left(x\left(t_{1}\right)\right) \leq \sum_{k=t_{1}}^{t_{2}} E(w, z, k), t_{2}>t_{1} \geq 0
$$

The above inequality formalizes the idea that a dissipative system is characterized by the property that the change of internal storage $V\left(x\left(t_{2}\right)\right)-V\left(x\left(t_{1}\right)\right)$ on any time interval $\left[t_{1}, t_{2}\right]$ will never exceed the amount of supply that flows into the system. Taking into account the stochastic nature of the system (7), we define the notion of dissipativity in the sense of expectation.

Assume that the system (7) is given together with a meansquare energy supply function $E(w, z, T)$ defined by

$$
\begin{aligned}
E(w, z, T)= & \mathbf{E}\left\{\sum_{k=0}^{T} z^{T}(k) Q z(k)\right\}+2 \mathbf{E}\left\{\sum_{k=0}^{T} z^{T}(k) S z(k)\right\} \\
& +\mathbf{E}\left\{\sum_{k=0}^{T} w^{T}(k) R w(k)\right\},
\end{aligned}
$$

where $Q=Q^{T}, R=R^{T}, S$ with appropriate dimensions. Definition 3 . The closed-loop system (7) with the meansquare energy supply function $E(w, z, T)$ defined in (10), storage function $V(T)$, is said to be strictly mean-square $(Q, R, S)$-dissipative, under zero initial state, for any $T \geq 0$ and $w(k) \in l_{2}[0, T]$, if for some scalar $\alpha>0$, such that

$$
V(T)+\alpha \sum_{k=0}^{T} w^{T}(k) w(k)<E(w, z, T) .
$$

With these definitions, the objective of this paper is to design the output feedback controller (6) for the system (1), such that for both measurement and control packet dropouts, the closed-loop system (7) satisfies the following requirements i) The closed-loop system (7) is asymptotically meansquare stable with $w(k)=0$;

ii) Under the zero-initial condition, the system (7) is strictly mean-square $(Q, R, S)$-dissipative.

Throughout this paper, we need the following assumptions.

Assumption 1. Real matrices $\bar{L}, \bar{M}$ and $\bar{G}$ satisfy

$$
\left\{\begin{array}{l}
\hat{M}=\bar{L}+\bar{M} E_{3}+E_{3}^{T} \bar{M}^{T}+E_{3}^{T} \bar{G} E_{3}<0 \\
\bar{G} \geq 0
\end{array}\right.
$$

Assumption 2. Real matrices $R, Q, S$ are satisfied the following conditions

$$
\left\{\begin{array}{l}
R=R+D_{11}^{T} S+S^{T} D_{11}+D_{11}^{T} Q D_{11}>0 \\
Q_{-}=-Q>0 .
\end{array}\right.
$$

\subsection{Dissipative analysis}

Theorem 1. Given real matrices $\bar{L}, \bar{G}, \bar{M}$ satisfying Assumption 1 and $Q, R, S$ satisfying Assumption 2, with $Q, R, \bar{L}$, and $\bar{G}$ symmetric. Then under the zero initial state condition, the closed-loop system (7) is asymptotically mean-square stable and strictly mean-square $(Q, R, S)$-dissipative, if there exist positive definite matrices $P>0, W_{1}>0$ and $W_{2}>0$ such that

$$
\left[\begin{array}{ll}
\Psi_{1} & \Psi_{2}^{T} \\
\Psi_{2} & \Psi_{3}
\end{array}\right]<0
$$

where $a=\sqrt{\bar{\alpha}(1-\bar{\alpha})}, \quad b=\sqrt{\bar{\beta}(1-\bar{\beta})}$,

$$
\begin{aligned}
\Psi_{1} & =\left[\begin{array}{cccc}
-P & * & * & * \\
0 & -W_{2} & * & * \\
-S^{T} C_{c l 0} & -S^{T} C_{d} & -\hat{R} & * \\
\bar{M} E_{c l 0} & \bar{M} E_{d} & \bar{M} E_{2}-H_{2}^{T} S & \hat{M}
\end{array}\right], \\
\Psi_{3} & =\left[\begin{array}{cc}
\Lambda_{1} & \Lambda_{2}^{T} \\
\Lambda_{2} & \Lambda_{3}
\end{array}\right],
\end{aligned}
$$$$
\Psi_{2}=\left[\begin{array}{cccc}
P A_{c l 0} & P \hat{A}_{d} & P B_{c l 0} & P H_{1} \\
\sqrt{d} Z\left(A_{c l 0}-I\right) & \sqrt{d} Z \hat{A}_{d} & \sqrt{d} Z B_{c l 0} & \sqrt{d} Z H_{1} \\
E_{c l 0} & E_{d} & E_{2} & E_{3} \\
\sqrt{d} b Z A_{c l 2} & 0 & \sqrt{d} b Z B_{c l 2} & 0 \\
Q_{-} C_{c l 0} & Q_{-} C_{d} & Q_{-} D_{11} & Q_{-} H_{2} \\
b P A_{c l 2} & 0 & b P B_{c l 2} & 0 \\
a P A_{c l 1} & 0 & 0 & 0 \\
Z & 0 & 0 & 0 \\
a Q_{-} C_{c l 1} & 0 & 0 & 0 \\
\sqrt{d} a Z A_{c l 1} & 0 & 0 & 0 \\
a E_{c l 1} & 0 & 0 & 0
\end{array}\right],
$$

where

$$
\begin{aligned}
& \Lambda_{1}=\operatorname{diag}\left\{-P,-W_{1}^{-1},-\bar{G}^{-1},-W_{1}^{-1},-Q_{-},-P\right\}, \\
& \Lambda_{2}=\left[\begin{array}{lllllll}
0 & 0 & 0 & 0 & 0 & 0 \\
0 & 0 & 0 & 0 & 0 & 0 \\
0 & 0 & 0 & 0 & 0 & 0 \\
0 & 0 & 0 & 0 & 0 & 0
\end{array}\right], \\
& \Lambda_{3}=\operatorname{diag}\left\{-P,-W_{2}^{-1},-Q_{-},-W_{1}^{-1},-\bar{G}^{-1}\right\} .
\end{aligned}
$$

Proof. Denote

$$
\varepsilon(k)=\eta(k+1)-\eta(k)
$$

Since the parameters of system (7) contain the stochastic parameters, we separate the stochastic parameters as

$$
\begin{aligned}
& A_{c l}=A_{c l 0}+(\alpha-\bar{\alpha}) A_{c l 1}+(\beta-\bar{\beta}) A_{c l 2}, \\
& B_{c l}=B_{c l 0}+(\beta-\bar{\beta}) B_{c l 2}, \quad C_{c l}=C_{c l 0}+(\alpha-\bar{\alpha}) C_{c l 1}, \\
& E_{c l}=E_{c l 0}+(\alpha-\bar{\alpha}) E_{c l 1}
\end{aligned}
$$

where 


$$
\begin{aligned}
& A_{c l 0}=\left[\begin{array}{cccc}
A & \bar{\alpha} B_{2} & 0 & (1-\bar{\alpha}) B_{2} C_{F} \\
0 & \bar{\alpha} I & 0 & (1-\bar{\alpha}) C_{F} \\
(1-\bar{\beta}) C_{2} & 0 & \bar{\beta} I & 0 \\
(1-\bar{\beta}) B_{F} C_{2} & 0 & \bar{\beta} B_{F} & A_{F}
\end{array}\right], \\
& A_{c l 1}=\left[\begin{array}{cccc}
0 & B_{2} & 0 & -B_{2} C_{F} \\
0 & I & 0 & -C_{F} \\
0 & 0 & 0 & 0 \\
0 & 0 & 0 & 0
\end{array}\right], B_{c l 0}=\left[\begin{array}{c}
B_{1} \\
0 \\
(1-\bar{\beta}) D_{21} \\
(1-\bar{\beta}) B_{F} D_{21}
\end{array}\right] \text {, } \\
& A_{c l 2}=\left[\begin{array}{cccc}
0 & 0 & 0 & 0 \\
0 & 0 & 0 & 0 \\
-C_{2} & 0 & I & 0 \\
-B_{F} C_{2} & 0 & B_{F} & 0
\end{array}\right], B_{c l 2}=\left[\begin{array}{c}
0 \\
0 \\
-D_{21} \\
-B_{F} D_{21}
\end{array}\right], \\
& C_{c l 0}=\left[\begin{array}{llll}
C_{1} & \bar{\alpha} D_{12} & 0 & (1-\bar{\alpha}) D_{12} C_{F}
\end{array}\right], \\
& C_{c l 1}=\left[\begin{array}{lllll}
0 & D_{12} & 0 & -D_{12} C_{F}
\end{array}\right], \\
& E_{c l 0}=\left[\begin{array}{llll}
E_{1} & \bar{\alpha} E_{c} & 0 & (1-\bar{\alpha}) E_{c} C_{F}
\end{array}\right], \\
& E_{c l 1}=\left[\begin{array}{llll}
0 & E_{c} & 0 & -E_{c} C_{F}
\end{array}\right] .
\end{aligned}
$$

From (7), we can get that

$$
\begin{aligned}
\eta(k+1)= & \left(A_{c l}+\hat{A}_{d} Z\right) \eta(k)-\hat{A}_{d} Z \sum_{i=k-d}^{k-1} \varepsilon(i) \\
& +\left(B_{c l 0}+(\beta(k)-\bar{\beta}) B_{c l 2}\right) w(k) \\
& +\left(H_{c l 0}+(\beta(k)-\bar{\beta}) H_{c l 2}\right) \xi(k)
\end{aligned}
$$

and

$$
\begin{aligned}
\varepsilon(k)= & \left(A_{c l}-I+\hat{A}_{d} Z\right) \eta(k)-\hat{A}_{d} Z \sum_{i=k-d}^{k-1} \varepsilon(i) \\
& +\left(B_{c l 0}+(\beta(k)-\bar{\beta}) B_{c l 2}\right) w(k) \\
& +\left(H_{c l 0}+(\beta(k)-\bar{\beta}) H_{c l 2}\right) \xi(k) .
\end{aligned}
$$

Then choose a Lyapunov function

$$
\begin{aligned}
V(k)= & \eta^{T}(k) P \eta(k)+\sum_{i=k-d}^{k-1} \eta^{T}(i) Z^{T} W_{2} Z \eta(i) \\
& +\sum_{l=-d+1}^{0} \sum_{i=k-l-1}^{k-1} \varepsilon^{T}(i) Z^{T} W_{1} Z \varepsilon(i)
\end{aligned}
$$

To establish dissipative performance, we assume the zero initial state condition $V(0)=0$. For $T>0$, we introduce the cost function

$$
\begin{aligned}
J(T)= & \sum_{k=0}^{T} z(k)^{T} Q z(k)+2 \sum_{k=0}^{T} z^{T}(k) S w(k) \\
& +\sum_{k=0}^{T} w^{T}(k) R w(k) .
\end{aligned}
$$

Using Schur complement if (14) is satisfied, then $J(T)-$ $V(T)>0$. So the closed-loop networked control system (7) is asymptotically mean-square stable and strictly meansquare $(Q, R, S)$-dissipative, and the proof is completed.

\subsection{Dissipative control}

This section is devoted to designing the controller such that the closed-loop networked control system in (7) is asymptotically mean-square stable and strictly meansquare $(Q, S, R)$-dissipative.

First, we define

$$
\begin{array}{rlrl}
\breve{A}_{11} & =\left[\begin{array}{ccc}
A & \bar{\alpha} B_{2} & 0 \\
0 & \bar{\alpha} I & 0 \\
(1-\bar{\beta}) C_{2} & 0 & \bar{\beta} I
\end{array}\right], \breve{A}_{12}=\left[\begin{array}{c}
(1-\bar{\alpha}) B_{2} \\
(1-\bar{\alpha}) I \\
0
\end{array}\right], \\
\bar{A}_{11} & =\left[\begin{array}{ccc}
0 & 0 & 0 \\
0 & 0 & 0 \\
-C_{2} & 0 & I
\end{array}\right], & \bar{A}_{11} & =\left[\begin{array}{ccc}
0 & B_{2} & 0 \\
0 & I & 0 \\
0 & 0 & 0
\end{array}\right], \\
\bar{A}_{12} & =\left[\begin{array}{lll}
-B_{2}^{T} & -I & 0
\end{array}\right]^{T}, & \breve{A}_{21} & =\left[\begin{array}{lll}
(1-\bar{\beta}) C_{2} & 0 & \bar{\beta} I
\end{array}\right], \\
\overline{\bar{A}}_{21} & =\left[\begin{array}{lll}
-C_{2} & 0 & I
\end{array}\right], & \breve{B}_{1} & =\left[\begin{array}{lll}
B_{1}^{T} & 0 & (1-\bar{\beta}) D_{21}^{T}
\end{array}\right]^{T}, \\
\overline{\bar{B}}_{1} & =\left[\begin{array}{lll}
0 & 0 & -D_{21}^{T}
\end{array}\right]^{T}, & \breve{H} & =\left[\begin{array}{lll}
H_{1}^{T} & 0 & 0
\end{array}\right]^{T}, \\
\breve{C} & =\left[\begin{array}{lll}
C_{1} & \bar{\alpha} D_{12} & 0
\end{array}\right], & \bar{C} & =\left[\begin{array}{lll}
0 & D_{21} & 0
\end{array}\right], \\
\breve{E}_{0} & =\left[\begin{array}{llll}
E_{1} & \bar{\alpha} E_{c} & 0
\end{array}\right], & \breve{E}_{1} & =\left[\begin{array}{lll}
0 & E_{c} & 0
\end{array}\right], \\
\bar{A}_{d} & =\left[\begin{array}{lll}
A_{d}^{T} & 0 & 0
\end{array}\right]^{T}, & \bar{Z} & =\left[\begin{array}{lll}
I & 0 & 0
\end{array}\right] .
\end{array}
$$

Then the controller design problem is solved in the following theorem, and the controller parameters are given in terms of the solution to a matrix inequality, which can be solved by the extended cone complementarity linearization method.

Theorem 2. Given real matrices $\bar{L}, \bar{G}, \bar{M}$ satisfying Assumption 1 and $Q, R, S$ satisfying Assumption 2, with $Q, R, \bar{L}$, and $\bar{G}$ symmetric. Then the closed-loop system (7) is asymptotically mean-square stable and strictly meansquare $(Q, R, S)$-dissipative, if there exist positive definite matrices $N, U, V, K, T_{1}, T_{2}, \Gamma_{1}, \Gamma_{2}$ and $\Gamma_{3}$ such that

$$
\left[\begin{array}{cccc}
\Phi_{11} & * & * & * \\
\Phi_{21} & \Phi_{22} & * & * \\
\Phi_{31} & \Phi_{32} & \Phi_{33} & * \\
\Phi_{41} & \Phi_{42} & \Phi_{43} & \Phi_{44}
\end{array}\right]<0
$$

where

$$
\begin{aligned}
\Phi_{11} & =\left[\begin{array}{ccccc}
-N & * & * & * & * \\
-N & -U & * & * & * \\
0 & 0 & -T_{2} & * & * \\
\Upsilon_{41} & -S^{T} \breve{C} & -S^{T} C_{d} T_{2} & \Upsilon_{44} & * \\
\Upsilon_{51} & \bar{M} \breve{E}_{0} & \bar{M} E_{d} T_{2} & \bar{M} E_{2}-H_{2}^{T} S & \Upsilon_{55}
\end{array}\right], \\
\Phi_{21} & =\left[\begin{array}{ccccc}
\Upsilon_{61} & \breve{A}_{11} & \bar{A}_{d} T_{2} & \breve{B}_{1} & \breve{H} \\
\Upsilon_{71} & \Upsilon_{72} & \bar{A}_{d} T_{2} & \Upsilon_{74} & \breve{H} \\
\Upsilon_{81} & \Upsilon_{82} & \sqrt{d} \bar{Z} \bar{A}_{d} T_{2} & \sqrt{d} \bar{Z} \breve{B}_{1} & \sqrt{d} \bar{Z} \breve{H} \\
\Upsilon_{91} & \breve{E}_{0} & E_{d} T_{2} & E_{2} & E_{3} \\
\Upsilon_{101} & \Upsilon_{102} & 0 & \sqrt{d} b \bar{Z} \bar{B}_{1} & 0
\end{array}\right], \\
\bar{V} & =\left[\begin{array}{cc}
-V & * \\
-K & -K
\end{array}\right], \Phi_{22}=\operatorname{diag}\left\{\bar{V},-T_{1},-\bar{G}^{-1},-T_{1}\right\}, \\
\Phi_{31} & =\left[\begin{array}{ccccc}
\Xi_{11} & Q_{-} \breve{C} & Q_{-} C_{d} T_{2} & Q_{-} D_{11} & 0 \\
b \bar{A}_{11} & b \bar{A}_{11} & 0 & b \bar{B} & 0 \\
\Xi_{31} & \Xi_{31} & 0 & \Xi_{34} & 0 \\
\Xi_{41} & a \bar{A}_{11} & 0 & 0 & 0 \\
\Xi_{41} & a \bar{A}_{11} & 0 & 0 & 0
\end{array}\right],
\end{aligned}
$$




$$
\begin{aligned}
& \Phi_{32}=\left[\begin{array}{lllll}
0 & 0 & 0 & 0 & 0 \\
0 & 0 & 0 & 0 & 0 \\
0 & 0 & 0 & 0 & 0 \\
0 & 0 & 0 & 0 & 0 \\
0 & 0 & 0 & 0 & 0
\end{array}\right], \quad \Phi_{42}=\Phi_{43}=\left[\begin{array}{lllll}
0 & 0 & 0 & 0 & 0 \\
0 & 0 & 0 & 0 & 0 \\
0 & 0 & 0 & 0 & 0
\end{array}\right], \\
& \Phi_{33}=\operatorname{diag}\left\{-Q_{-}, \bar{V}, \bar{V}\right\}, \\
& \Phi_{41}=\left[\begin{array}{ccccc}
\bar{Z} & \bar{Z} & 0 & 0 & 0 \\
a Q_{-}\left(\bar{C}-D_{12} \Gamma_{3}\right) & a Q_{-} \bar{C} & 0 & 0 & 0 \\
a \sqrt{d} \bar{Z}\left(\bar{A}_{11}+\bar{A}_{12} \Gamma_{3}\right) & a \sqrt{d} \bar{Z} \bar{A}_{11} & 0 & 0 & 0 \\
a\left(\breve{E}_{1}-E_{c} \Gamma_{3}\right) & a \bar{E}_{1} & 0 & 0 & 0
\end{array}\right], \\
& \Phi_{44}=\operatorname{diag}\left\{-T_{2},-Q_{-},-T_{1},-\bar{G}^{-1}\right\} \text {, } \\
& \Upsilon_{41}=-S^{T}\left(\breve{C}+(1-\bar{\alpha}) D_{21} \Gamma_{3}\right) \text {, } \\
& \Upsilon_{44}=-R-D_{11}^{T} S-S^{T} D_{11} \text {, } \\
& \Upsilon_{51}=\bar{M}\left(\breve{E}_{0}+(1-\bar{\alpha}) E_{c} \Gamma_{3}\right) \\
& \Upsilon_{55}=\bar{L}+\bar{M} E_{3}+E_{3}^{T} \bar{M}^{T}, \Upsilon_{61}=\breve{A}_{11}+\breve{A}_{12} \Gamma_{3} \text {, } \\
& \Upsilon_{71}=\breve{A}_{11}+\Gamma_{2} \breve{A}_{21}+\breve{A}_{12} \Gamma_{3}+\Gamma_{1} \text {, } \\
& \Upsilon_{72}=\breve{A}_{11}+\Gamma_{2} \breve{A}_{21}, \quad \Upsilon_{74}=\breve{B}_{1}+(1-\bar{\beta}) \Gamma_{2} D_{21}, \\
& \Upsilon_{81}=\sqrt{d} \bar{Z}\left(\breve{A}_{11}-I+\breve{A}_{12} \Gamma_{3}\right), \\
& \Upsilon_{82}=\sqrt{d} \bar{Z}\left(\breve{A}_{11}-I\right), \quad \Upsilon_{101}=\Upsilon_{102}=\sqrt{d} b \bar{Z} \overline{\bar{A}}_{11} \text {, } \\
& \Upsilon_{91}=\breve{E}_{0}+(1-\bar{\alpha}) E_{c} \Gamma_{3} \text {, } \\
& \Xi_{11}=Q_{-} \breve{C}+(1-\bar{\alpha}) Q_{-} D_{21} \Gamma_{3} \text {, } \\
& \Xi_{31}=b\left(\overline{\bar{A}}_{11}+\Gamma_{2} \overline{\bar{A}}_{21}\right), \quad \Xi_{33}=b\left(\overline{\bar{B}}_{11}-\Gamma_{2} D_{21}\right), \\
& \Xi_{35}=b\left(\bar{H}-\Gamma_{2} H_{3}\right), \quad \Xi_{41}=a\left(\bar{A}_{11}+\bar{A}_{12} \Gamma_{3}\right) \text {. }
\end{aligned}
$$

Moreover, the parameters of the controller are

$$
\left\{\begin{array}{l}
A_{F}=X_{12}^{-1} U \Gamma_{1} V Y_{12}^{-T}, \\
B_{F}=X_{12}^{-1} U \Gamma_{2}, \\
C_{F}=\Gamma_{3} V Y_{12}^{-T},
\end{array}\right.
$$

where $I-U V=X_{12} Y_{12}^{T}<0$.

Proof. Firstly, we partition $P$ and $P^{-1}$ as

$$
P=\left[\begin{array}{cc}
U & X_{12} \\
X_{12}^{T} & X_{22}
\end{array}\right], P^{-1}=\left[\begin{array}{cc}
V & Y_{12} \\
Y_{12}^{T} & Y_{22}
\end{array}\right],
$$

where the partition is compatible with the parameters of the closed-loop system.

Let $\Pi_{1}=\left[\begin{array}{cc}V & I \\ Y_{12}^{T} & 0\end{array}\right], \Pi_{2}=\left[\begin{array}{cc}I & U \\ 0 & X_{12}^{T}\end{array}\right]$, which imply that $P \Pi_{1}=\Pi_{2}$ and $\Pi_{1}^{T} P \Pi_{1}=\Pi_{1}^{T} \Pi_{2}$.

We apply the congruence transformation

$$
\operatorname{diag}\left\{\Pi_{1}, I, I, I, \Pi_{1}, I, I, I, I, \Pi_{1}, \Pi_{1}, I, I, I, I\right\}
$$

to $(14)$.

Define that $V=N^{-1}, K=U^{-1}, T_{1}=W_{1}^{-1}$ and $T_{2}=W_{2}^{-1}$.

Then apply the congruence transformation

$$
\operatorname{diag}\{N, I, I, I, I, I, K, I, I, I, I, I, K, I, K, I, I, I, I\}
$$

again. After that, we can obtain (20).
The formula(20)implies that: $I-U V=X_{12} Y_{12}^{T}<0$. So if there exist the nonsingular matrices $X_{12}$ and $Y_{12}$, the controller exists. Based on Theorem 1, system (7) is meansquare stable and satisfies the dissipative performance, which completes the proof.

Theorem 2 gives the design method of the controller, but it should satisfy the equation constraints

$$
V=N^{-1}, \quad K=U^{-1}
$$

For $N>0, V>0$, and $\left[\begin{array}{cc}N & I \\ I & V\end{array}\right]>0$, then we can obtain that $V N=I$ if and only if $\operatorname{trace}(V N) \geq n+m+p$. Similarly, $\operatorname{trace}(K U) \geq n+m+p$.

It is noted that this condition is not a convex set for the matrix equality constraints. This problem can be solved via the cone complementarity linearization method described by Ghaoui et al. (1997) or the sequential linear programming matrix method, which was proposed by Leibfritz (2001). But both of them are defective. The cone complementarity linearization method is highly conservative. Although the sequential linear programming matrix method has improved the cone complementarity linearization method, it also exists defect. It can not realize the iteration between 0 and 1 . Because when $\rho=0$, the iteration stops. The algorithm proposed in this paper avoids this occurrence.

This paper proposes the extended cone complementarity linearization method as follows

Step 1: Given the maximum iteration times $N$, and the iteration accuracy $\epsilon$;

Step 2: Find the feasible solution to (20), (23), and let $N^{(k)}=N, V^{(k)}=V, K^{(k)}=K, U^{(k)}=U, k=0$;

Step 3: Find a set of optimal solution $\hat{N}, \bar{U}, \tilde{V}, W_{1}, W_{2}, \Gamma_{1}$, $\Gamma_{2}$ and $\Gamma_{3}$, such that mintrace $\left(N V^{(k)}+N^{(k)} V+\right.$ trace $\left(K U^{(k)}+K^{(k)} U\right)$, subject to $(20)$;

Step 4: If the formula (23) is satisfied, and $\mid \operatorname{trace}\left(N V^{(k)}+\right.$ $\left.N^{(k)} V\right)+\operatorname{trace}\left(K U^{(k)}+K^{(k)} U\right)-4(n+m+p) \mid \leq \epsilon$; else Stop.

Step 5: If $k>N$, then output the optimal solutions. Iteration stops; else go to Step 6 ;

Step 6: Let $N^{(k)}=N, V^{(k)}=V, K^{(k)}=K, U^{(k)}=$ $U$ and $k=k+1$, go to Step 3 .

Remark 1. We will consider both network-induced delays and packet dropouts in the following paper.

\section{A NUMERICAL EXAMPLE}

In this section, we give an example to demonstrate the effectiveness of the proposed method. For this purpose, consider the model as the system (1) with parameters

$$
A=\left[\begin{array}{ccc}
0.9995 & -0.8339 & 0.09976 \\
0.3032 & 0.9894 & -0.2667 \\
-0.01008 & -0.0166 & 0.9949
\end{array}\right], A_{d}=\left[\begin{array}{ccc}
-0.20 .1 & 0 \\
0 & 0.1 & 0 \\
0.5 & 0 & 0.05
\end{array}\right],
$$




$$
\begin{aligned}
H_{1} & =\left[\begin{array}{lll}
0.5 & 0.5 & 1
\end{array}\right]^{T}, \quad B_{1}=\left[\begin{array}{lll}
0.5882 & 0.7151 & 0.01175
\end{array}\right]^{T}, \\
B_{2} & =\left[\begin{array}{lll}
0.7387 & -0.03757 & 0.01479
\end{array}\right]^{T}, \\
C_{1} & =\left[\begin{array}{lll}
0.018 & 0.114 & 0.47
\end{array}\right], \quad C_{2}=\left[\begin{array}{lll}
0.5 & 0.6 & 0.3
\end{array}\right], \\
C_{d} & =\left[\begin{array}{lll}
0.023 & 0.114 & 0.43
\end{array}\right], \quad E_{1}=\left[\begin{array}{lll}
0.1 & 0 & 0
\end{array}\right], \\
D_{11} & =0.1, \quad D_{12}=1, \quad D_{21}=0.1, \\
H_{2} & =0.005, \quad H_{3}=0.5, \quad E_{2}=0.1, \quad E_{3}=0.1, \\
E_{4} & =0.1, \quad E_{c}=0.2, \quad E_{d}=\left[\begin{array}{lll}
0.1 & 0 & 0
\end{array}\right], \quad d=1 .
\end{aligned}
$$

Matrices $Q, S, \bar{L}, \bar{G}$ and $\bar{M}$ are given by $Q=-1, S=0, R=\gamma^{2} I, \bar{L}=-0.1, \bar{G}=0.2, \bar{M}=0.1$. Assume that $\operatorname{Prob}\{\beta(k)=1\}=0.9$ and $\operatorname{Prob}\{\alpha(k)=$ $1\}=0.9$. From (20), using extended cone complementarity linearization method and MATLAB LMI toolbox, we can obtain the parameters of the controller are

$$
\begin{aligned}
& A_{F}=\left[\begin{array}{ccccc}
-0.1119 & 0.0571 & 0.0230 & -2.0070 & -0.0095 \\
-0.0324 & -0.0541 & 0.0381 & 0.3161 & -0.0249 \\
-0.0019 & -0.0122 & 0.0022 & -1.8495 & -0.0025 \\
0.0025 & 0.0000 & -0.0017 & 0.7819 & -0.0001 \\
-0.0009 & -0.0068 & 0.0060 & -2.9648 & 0.0008
\end{array}\right], \\
& B_{F}=\left[\begin{array}{lllll}
-0.0179 & -0.0338 & -0.0603 & -0.0002 & -0.9932
\end{array}\right]^{T} \text {, } \\
& C_{F}=\left[\begin{array}{lllll}
-0.0080 & 0.0063 & 0.0010 & -0.8710 & -0.0001
\end{array}\right] \text {. }
\end{aligned}
$$

The table next shows the performance of the system with different probabilities of packet dropouts.

\begin{tabular}{|c||c||c|}
\hline $\bar{\alpha}$ & $\bar{\beta}$ & $\gamma$ \\
\hline 0.9 & 0.9 & 1.1879 \\
\hline 0.9 & 0.5 & 1.1782 \\
\hline 0.5 & 0.5 & 1.1538 \\
\hline 0.2 & 0.5 & 1.1298 \\
\hline \hline 0.2 & 0.2 & 1.1242 \\
\hline
\end{tabular}

Table 1. The $\overline{\overline{\text { cases with different }}}$ probabilities

From what we said above we can know that, the more packet dropouts, the poorer the performance of the system.

\section{CONCLUSION}

This paper has addressed the dissipative output feedback control for uncertain time-delay networked control systems. The Bernoulli distributed sequences have been used to describe the multiple measurement and control packet dropouts. The dissipativity performance condition has been proposed in the form of linear matrix inequality. Based on this condition, a controller design procedure has been developed, which guarantees the closed-loop NCS is asymptotically mean-square stable and strictly meansquare $(Q, S, R)$-dissipative. Then the controller gain has been obtained by using the extended cone complementarity linearization method, which is better than a sequential linear programming matrix method. The relationship between robust dissipative control and uncertain time-delay networked control systems has been also established for the first time. The illustrative example has been provided to present the effectiveness of the proposed design method.

\section{REFERENCES}

J. P. Hespanha, P. Naghshtabrizi and Y. Xu. A survey of recent results in networked control systems. Proceedings of IEEE, 95 (1): 138-162, 2007.

W. Zhang, M. S. Branicky and S. M. Phillips. Stability of networked control systems. IEEE Control Systems Magazine, 21: 84-99, 2001.

D. Yue, Q.-L. Han and C. Peng. State feedback controller design of networked control systems. IEEE Transactions on Circuits and Systems-II: Express Briefs, 51 (11): 640644, 2004.

M. Yu, L. Wang, T. Chu and F. Hao. An LMI approach to networked control systems with data packet dropout and transmission delays. Proceedings of the 43 rd IEEE conference on Decision and Control, pages 3545-3550, 2004

J. C. Willems. Dissipative dynamical systems-part I: General theory. Archives for the Rational Mechanics and Analysis, 45: 321-393, 1972.

S. Xie, L. Xie, C. E. De Souza. Robust dissipative control for linear systems with dissipative uncertainty. International Journal of Control, 70 (2): 169-191, 1998.

Z. Li, J. Wang and H. Shao. Delay-dependent dissipative control for linear time-delay systems. Journal of the Franklin Institute, 339: 529-542, 2002.

L. Yang, Q. L. Zhang, G. Y. Liu and P. Y. Liu. Robust impulse dissipative control of singular systems with uncertainties. Acta Automatica Sinica, 33 (5): 554-556, 2007.

T. Shen, K. Tamura. Robust tracking control for robot systems: A dissipative systems theory approach. Transactions of the Society of Instrument and Control Engineers, 35 (3): 326-332, 1999.

H. Zhang, Z. H. Guan and G. Feng. Reliable dissipative control for stochastic impulsive systems. Automatica, 44 (4): 1004-1010, 2008.

H. Gao, T. Chen and T. Chai. Passivity and passification for networked control systems. SIAM Journal of Control and Optimization, 46 (4): 1299-1322, 2007.

R. Lozano, B. Brogliato, O. Egeland and B. Maschke. Dissipative systems analysis and control: Theory and appliactions. Springer-Verlag, London, 2000.

El. Ghaoui, F. Oustry, and M. A. Rami. A cone complementarity linearization algorithm for static outputfeedback and related problems. IEEE Transactions on Automatic Control, 42 (8): 1171-1176, 1997.

F. Leibfritz. An LMI-based algorithm for designing suboptimal static $\mathrm{H}_{2} / \mathrm{H}_{\infty}$ output feedback controllers. SIAM Journal of Control and Optimization, 39 (6): 17111735,2001 